\title{
Design and Performance Analysis of MISO-SRMR-DCSK System over Rayleigh Fading Channels
}

\author{
Gang Zhang, Yi man Hao $\mathbb{D}$, and Tian qi Zhang \\ School of Communication and Information Engineering, Chongqing University of Posts and Telecommunications, \\ Chongqing 400065, China \\ Correspondence should be addressed to Yi man Hao; hao_yiman@foxmail.com
}

Received 30 January 2018; Accepted 14 May 2018; Published 13 August 2018

Academic Editor: Giuseppina Monti

Copyright (c) 2018 Gang Zhang et al. This is an open access article distributed under the Creative Commons Attribution License, which permits unrestricted use, distribution, and reproduction in any medium, provided the original work is properly cited.

\begin{abstract}
The major drawback of the differential chaos shift keying (DCSK) system is that equal time and energy are spent on the reference and data signal. This paper presents the design and performance analysis of a short reference multifold rate DCSK (SRMR-DCSK) system to overcome the major drawback. The SRMR-DCSK system is proposed to enhance the data rate of the short reference differential chaos shift keying (SR-DCSK) system. By recycling each reference signal in SR-DCSK, the data slot carries $\mathrm{N}$ bits of data and by $\mathrm{P}$ times. As a result, compared with SR-DCSK, the proposed system has a higher data transmission rate and evaluates the energy efficiency with respect to the conventional DCSK system. To further improve the bit-error-rate (BER) performance over Rayleigh fading channels, the multiple-input single-output SRMR-DCSK (MISO-SRMR-DCSK) is also studied. The BER expression of the proposed system is derived based on Gaussian approximation (GA), and simulations in Rayleigh fading channels are performed. Simulation results show a perfect match with the analytical expression.
\end{abstract}

\section{Introduction}

CHAOS signals are suitable for spread spectrum communications due to broadband and nonperiodic characteristics. In the past few decades, the coherent and noncoherent algorithms of various chaotic communication systems have been proposed [1-15]. Coherent detection requires synchronization of chaotic carriers, for example, chaos shift keying (CSK) [1]; therefore, its application is limited. In light of the shortcomings of coherent detection, differential chaos shift keying (DCSK) [2] becomes the most worthy consideration in the development of chaotic communication system. The reason is that the DCSK system can demodulate the transmitted information at the receiver without the chaotic carrier synchronization and channel state estimation. In addition, DCSK has better antimultipath fading capability than differential phase shift keying (DPSK) [3] and is more suitable for ultra-wideband transmission. However, the traditional DCSK system transmission efficiency is low, and low security limits its application. To overcome the shortcoming of traditional DCSK, researchers have proposed a number of improvements. For example, in [4], Herceg et al. introduce the correlation multidelay shift keying (CMDCK). In their proposal, the reference slot and the data slot that delays different chip times are subtracted to transmit the multibit information to improve the data rate. In [5], quadrature chaos-shift keying (QCSK), an orthogonal chaotic keying system, is proposed by Galias and Maggio through the Hilbert transformation to obtain two completely orthogonal chaotic sequences for the transmission of two consecutive time slots. This improvement of transmission rate is sacrificed at the expense of the cost of modulation. In [6], Kaddoum et al. propose the short reference DCSK (SRDCSK) system, a work in which the length of the reference slot is shortened while the data slot remains unchanged. Moreover, Cai et al. propose M-ary DCSK [7] and differential DCSK [8], which are also aimed at improving the DCSK transmission rate. Kaddoum et al. [9] introduced a multicarrier DCSK system to improve the safety of the DCSK system, and [10] propose a new MC-DCSK system, where no RF delay line is required, while improving the spectrum utilization and reducing energy consumption. In [11], Xu et al. 
propose the code-shifted DCSK (CS-DCSK), which makes the Walsh code to distinguish between reference slot and data slot, thereby reducing the interference between the signal and improving the performance of BER. The extension of CS-DCSK is proposed in [12], in which $\mathrm{N}$ bits of information are transmitted in the same time slot, while using only a reference slot and obtaining a high transmission rate. In [13], high-efficiency DCSK (HE-DCSK) is studied and inspected. In this system, the data signal transmits 2 bits of data, where the 1-bit signal is demodulated by the reference slot of the previous frame. And it improves the utilization of the reference slot. In [14], reference-modulated DCSK (RM-DCSK) is introduced. RM-DCSK is to use the previous frame as the reference signal of the latter frame to improve the data transmission efficiency. The permutation DCSK (P-DCSK) proposed in [15] is to add the random permutation matrix in the traditional DCSK system to disrupt the sequence of chaotic signal samples, destroy the similarity between the reference signal and the information signal, and eliminate the possibility of detecting the bit rate from the square spectrum, thereby increasing the data security. However, P-DCSK does not improve the DCSK system data transmission rate.

In this work, we propose a novel DCSK modulation scheme based on the SR-DCSK system, named short reference multifold rate differential chaos shift keying (SRMR-DCSK). In this system, a data frame is composed of the reference slot and the data slot, and the data slot can transmit $N$ bits of data at a time. And the length of the reference slot is $1 / P$ of the data slot, where $P$ is any integer that can be divisible by a spreading factor. This solution solves the problem that the DCSK reference slot does not transmit information. In order to improve the BER performance of the system over the Rayleigh fading channels, the multiple-input single-output SRMR-DCSK (MISO-SRMRDCSK) is proposed. In the paper, we define $M$ as the number of transmit antennas and $S$ the number of receive antennas, where $M=2$ and $S=1$. As a potential application, the MISO-SRMR-DCSK is considered and the BER formula is derived over the Rayleigh fading channel.

The rest of this paper is organized as follows: In Section 2, a description of the SISO-SRMR-DCSK transmitter scheme and demodulation structure is given, and the traditional DCSK system is described briefly. Moreover, the energy efficiency and data rate of SISO-SRMR-DCSK are analyzed and the energy efficiency is evaluated with respect to the conventional DCSK system. In Section 3, SISO-SRMR-DCSK is extended to MISO-SRMR-DCSK and analytical BER expression is explained. MISO-SRMR-DCSK, the extension of the SISO-SRMR-DCSK, is explained. The performance of the MISO-SRMR-DCSK system is derived in Gaussian approximation (GA). Section 4 simplifies the channel model between each antenna and the receiving antenna at the transmitter to a two-way Rayleigh fading channel. Further, it is assumed that the channel is slowly fading; that is, the channel is static in a one-bit period. If the gain of one channel is zero and the other gain is 1 , it is the Gaussian channel. The simulation of the new system is compared with other systems in Section 4. And the conclusion is presented in Section 5 .

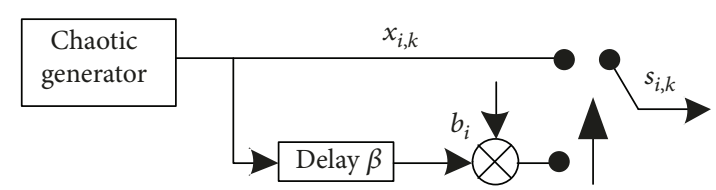

FIGURE 1: DCSK transmitter.

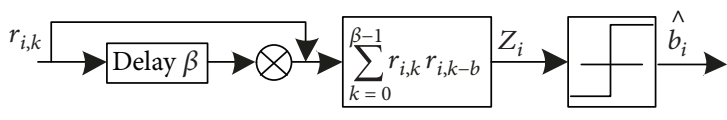

FIGURE 2: DCSK receiver.

\section{Noncoherent Chaos-Based Communication System}

2.1. DCSK System. In the modulation of the DCSK system, each bit represents the same length of two chaotic sequences in the frame. The first time slot transmits a chaotic sequence as the reference sequence, and the second one transmits the chaotic sequence of the modulated data as data sequence. If +1 is transmitted, the reference sequence is equal to the data sequence, and if -1 is transmitted, the reverse reference sequence is transmitted as a data sequence. The spreading factor in the DCSK system is defined as the number of chaotic samples used to spread each transmitted bit and is presented by $2 \beta$, where $\beta$ is an integer. In addition, the bit period of the DCSK system is $T_{\text {DCSK }}=$ $2 T_{b}=2 \beta T_{c}$, where $T_{c}$ is the chip time and $T_{c}=1$, and $T_{b}$ is a slot time, that is, half a bit of time. The $i$ th bit interval at the output of the DCSK system modulator can be expressed as (1) below.

$$
s_{i, k}= \begin{cases}x_{i, k}, & 0<k \leq \beta \\ b_{i} x_{i, k-\beta}, & \beta<k \leq 2 \beta,\end{cases}
$$

where $x_{i, k}$ is the chaotic sequence of the reference signal, $x_{i, k-\beta}$ is the delay signal of $x_{i, k}$, and $k$ represents any one of the chips in a frame. In order to demodulate the transmitted information, the received signal will be compared with its delay signal for the length of time $T_{b}\left(T_{b}=\beta T_{c}\right)$ correlation calculation, and it is determined by the zero threshold in the receiver. The transmitter structure and receiver structure are given in Figures 1 and 2, respectively. In Figure 1, for every bit of information, the transmitter outputs a chaotic sequence $x_{i, k}$ of length $\beta$ followed by the same sequence multiplied by the information signal $b_{i}= \pm 1$, where $i$ is the bit counter. In Figure 2, the received signal $r_{i, k}$ is multiplied by the received signal delayed by $\beta, r_{i, k-\beta}$. The product is then averaged over the spreading sequence length $\beta$. In the DCSK system, half of the frame time is used to transmit the reference signal that does not carry any useful information; thus, the data transmission rate is reduced.

2.2. SISO-SRMR-DCSK System. In the SISO-SRMR-DCSK system, the length of the reference signal and the length of 


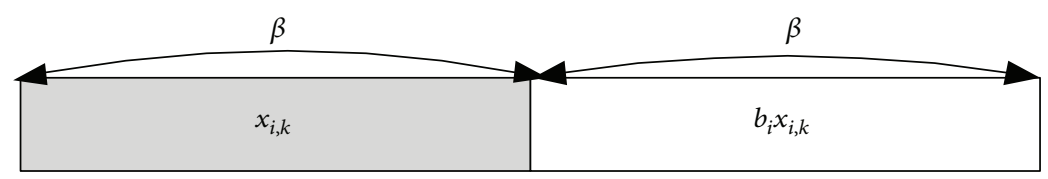

(a) DCSK frame

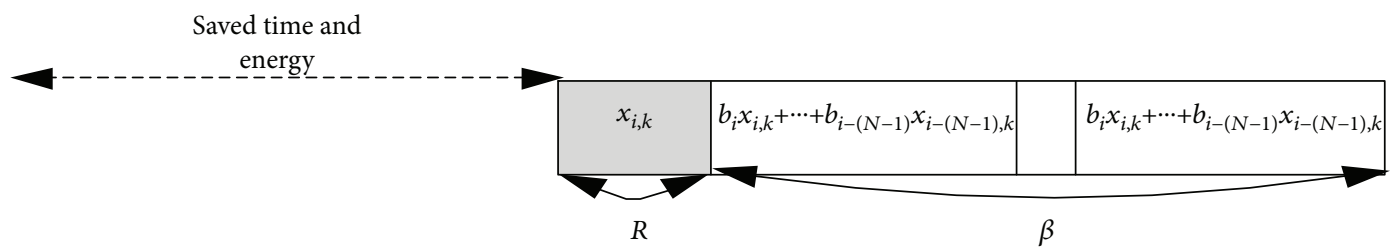

(b) SISO-SRMR-DCSK frame

FIGURE 3: DCSK frame and SISO-SRMR-DCSK frame.

the data signal are different. This is different from the traditional DCSK. For the SISO-SRMR-DCSK system, the length of the reference signal changes from $\beta$ to $R$, where $R$ is $1 / P$ of the data signal length. Meanwhile, the reference signal is repeatedly transmitted by $P$ times and carries $N$ bits of information as the data signal. The reference signal is correlated with each section of the data signals. The length of one frame changes from $2 \beta$ to $R+\beta=R+P \cdot R$. By comparing Figures $3(\mathrm{a})$ and $3(\mathrm{~b})$, we can see that the SISO-SRMR-DCSK system improves the data transmission rate and reduces the bit energy compared to the DCSK system.

Figure 4 shows the transmitter of the SISO-SRMR-DCSK system. The principle of the SISO-SRMR-DCSK system is that the reference signal of the previous bit is modulated and then added to the data signal of the latter bit in the data slot of the latter bit while reducing the length of the reference signal. The method reduces the wastage that any information is not carried in the reference signal. Take the signal transmitted by the $i$ th frame as an example. In the transmitter, the chaotic sequence of length $R$ is generated by the chaos generator as the reference sequence of this frame and then the chaotic sequence of length $R$ through the repeater. The output of the repeater is modulated by the different delay devices on the corresponding chaotic sequence, and the $N$ channel-modulated chaotic sequence is summed together by an adder as the data signal of the $i$ th frame. The $i$ th bit interval at the output of the SISO-SRMR-DCSK system modulator can be expressed as (2) below:

$$
s_{i, k}=\left\{\begin{array}{cc}
x_{i, k} & 0 \leq k<R, \\
b_{i} x_{i, k-R}+\cdots+b_{i-(N-1)} x_{i-(N-1), k-[(N-1) P+N] R} & R \leq k<\beta, \\
& x_{i, k-R} \equiv x_{i, 0}, \bmod (R),
\end{array}\right.
$$

where $x_{i, k}$ is the chaotic sequence of the reference signal and $x_{i, k-R}, \ldots, x_{i-(N-1), k-[(N-1) P+N] R}$ is the delay signal of $x_{i, k}$.

For the design of the frame structure, the duration of the corresponding frame is changed from $T_{\text {DCSK }}=2 \beta T_{c}$ to $T_{\text {SISO-SRMR-DCSK }}=(R+\beta) T_{c}$. The SISO-SRMR-DCSK system

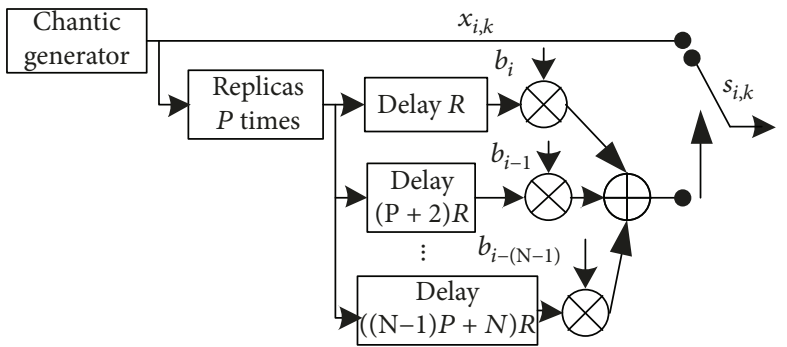

FIGURE 4: SISO-SRMR-DCSK transmitter.

compared to the DCSK system to improve the data transmission rate can be expressed below:

$$
R_{D}=\frac{R_{\text {SISO-SRMR-DCSK }}-R_{\mathrm{DCSK}}}{R_{\mathrm{DCSK}}} \times 100 \%,
$$

where $R_{D}$ is the increase percentage that the transmission rate of the SRMR-DCSK system is compared with the conventional DCSK system, and $R_{\text {SISO-SRMR-DCSK }}=N / R+\beta$ and $R_{\mathrm{DCSK}}=1 / 2 \beta$ are the data transmission rate of the SRMR-DCSK system and DCSK system and substituted into (3), respectively.

The above would be simplified to (4) below:

$$
R_{D}=\frac{(2 N-1) \beta-R}{\beta+R} \times 100 \%
$$

The SRMR-DCSK system can be used to describe the bit energy savings compared to the DCSK system in the same way:

$$
E_{D}=\frac{E_{b, \mathrm{DCSK}}-E_{b, \text { SISO-SRMR-DCSK }}}{E_{b, \mathrm{DCSK}}} \times 100 \%,
$$

where $E_{D}$ is the increase percentage that the saved bit energy of the SRMR-DCSK system is compared with the conventional DCSK system. 


$$
E_{b, \mathrm{DCSK}}=2 \beta T_{c} E\left[x_{k}^{2}\right] \text {, }
$$

$E_{b, \text { SISO-SRMR-DCSK }}$

$$
\begin{aligned}
=\frac{1}{N}\left[\sum_{k=0}^{R-1} x_{i, k}^{2}+\sum_{k=\mathrm{R}}^{\beta+\mathrm{R}}\left(b_{i} x_{i, k-R}+b_{i-1} x_{i-1, k-(P+1) R}+\cdots\right.\right. \\
\left.\left.+b_{i-(N-1)} x_{i-(N-1), k-[(N-1) P+N] R}\right)^{2}\right] T_{c},
\end{aligned}
$$

which would simplify to

$$
E_{b, \text { SISO-SRMR-DCSK }}=\frac{1}{N}(N \beta+\mathrm{R}) T_{c} E\left[x_{k}^{2}\right] .
$$

Equations (6) and (8) are substituted into (5):

$$
E_{D}=\frac{N \beta-R}{2 N \beta} \times 100 \% .
$$

The simulation curve in Figure 5 is obtained by (9). Figure 5 shows the bit energy enhancement percentages of SRMR-DCSK compared to DCSK for different values of $R$ when the spreading factor $\beta=100$. As can be seen from Figure 5, reducing the length of the reference signal can significantly improve the transmission rate, and increasing the number of transmitted bits can further increase the data transmission rate. As the number of transmitted bits increases, the rate of data transmission increases gradually close to $50 \%$.

Figure 6 shows the receiver of the SRMR-DCSK system. The principle of the receiver is that the received signal is correlated with the signals after the delay different times, and the outcome is compared to the zero threshold. In Figure 6, the receiver employs $N$ independent correlation branches to decode the $N$-bit data signal in the second slot; that is, the received signal $r_{i, k}$ is multiplied by the received signal delayed by $R,(P+2) R, \ldots,[(N-1) P+N] R$. The product is then averaged over the spreading sequence length $R$.

\subsection{MISO-SRMR-DCSK System. As the MISO-SRMR-DCSK} system, there are two transmission antennas $\left(N_{T}=2\right)$ in the transmitter and one receiving antenna in the receiver. So the receiver is the same as the SRMR-DCSK receiver.

2.4. Channel Model. In the text, each antenna uses two independent paths of Rayleigh quasi-static block fading channels; the channel model is shown in Figure 7.

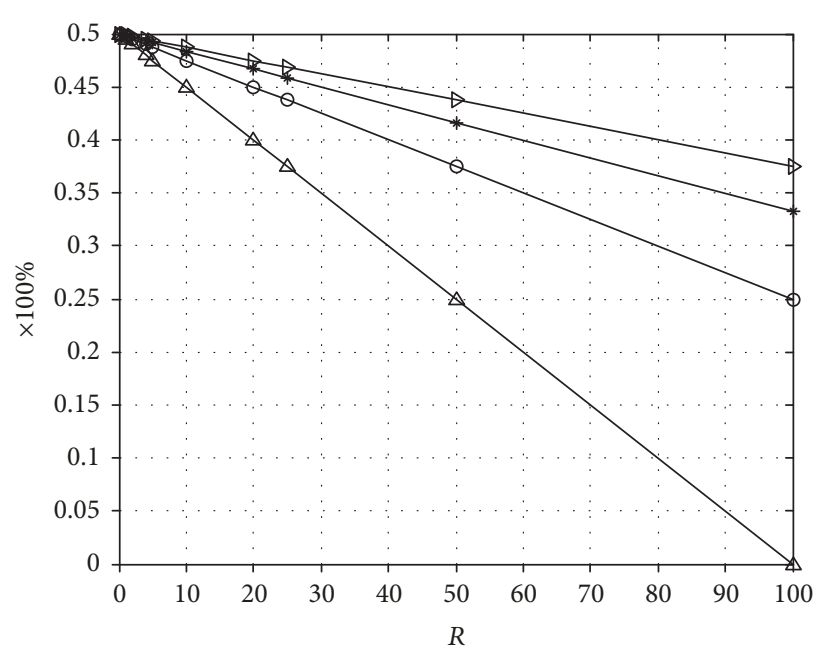

$$
\begin{array}{ll}
\triangle & N=1 \\
\square & N=2 \\
\longrightarrow * & N=3 \\
\longrightarrow & N=4
\end{array}
$$

FIGURE 5: Bit energy enhancement percentages of SRMR-DCSK compared to DCSK for the spreading factor $\beta=100$.

$\alpha_{n, 1}$ and $\alpha_{n, 2}$ are the channel coefficients following Rayleigh distribution, and $\xi_{i}$ is the Gaussian noise, which follows Gaussian distribution with zero mean and variance $N_{0} / 2$.

At the receiver, the receiver signal can be given by

$$
r_{i, k}=\sum_{n=1}^{N_{T}} \alpha_{n, 1} s_{i, k}+\sum_{n=1}^{N_{T}} \alpha_{n, 2} s_{i, k-\tau}+\xi_{i} .
$$

\section{Performance Analysis}

In this section, the performance of the MISO-SRMR-DCSK system is analyzed on the AWGN channel and the multipath Rayleigh fading channel. The mean and variance of this system are derived. In the text, the normalized logistic chaotic map is adopted, that is, $E[x]=0$ and $E\left[x^{2}\right]=\operatorname{var}[x]=1$, where $E[x]$ denotes the mean operator and var $[x]$ denotes the variance operator. Moreover, the signal components of (13) are independent of each other. And the chaotic sequence is independent of the Gaussian noise as well.

The decision variable $Z_{i-(m-1)}$ (for the sake of generality $m \geq 1$ ) output by the correlator in Figure 6 can be expressed as

$$
\begin{aligned}
& D_{i-(m-1)}=\sum_{k=0}^{R-1}\{ {\left[\frac{1}{\sqrt{N_{T}}}\left(\sum_{n=1}^{N_{T}} \alpha_{n, 1} x_{i-(m-1), k-[(m-1) P+m] R}+\sum_{n=1}^{N_{T}} \alpha_{n, 2} x_{i-(m-1), k-[(m-1) P+m] R-\tau}\right)+\xi_{i-(m-1), k-[(m-1) P+m] R}\right] } \\
&\left.\times\left[\frac{1}{\sqrt{N_{T}}}\left(\sum_{n=1}^{N_{T}} \alpha_{n, 1} \sum_{j=0}^{N-1} b_{i-j} x_{i-j, k-(j P+j+1) R}+\sum_{n=1}^{N_{T}} \alpha_{n, 2} \sum_{j=0}^{N-1} b_{i-j} x_{i-j, k-(j P+j+1) R-\tau}\right)+\xi_{i, k}\right]\right\} \\
& Z_{i-(m-1)}=\sum_{p=1}^{P} D_{i-(m-1)},
\end{aligned}
$$




$$
\begin{aligned}
Z_{i-(m-1)}= & \frac{P}{N_{T}} \sum_{k=0}^{R-1} \sum_{n=1}^{N_{T}} \alpha_{n, 1}^{2} x_{i-(m-1), k-[(m-1) P+m] R} \cdot \sum_{j=0}^{N-1} b_{i-j} x_{i-j, k-(j P+j+1) R}+\frac{P}{N_{T}} \sum_{k=0}^{R-1} \sum_{n=1}^{N_{T}} \alpha_{n, 1} x_{i-(m-1), k-[(m-1) P+m] R} \\
& \cdot \sum_{n=1}^{N_{T}} \alpha_{n, 2} \sum_{j=0}^{N-1} b_{i-j} x_{i-j, k-(j P+j+1) R-\tau}+\frac{P}{N_{T}} \sum_{k=0}^{R-1} \sum_{n=1}^{N_{T}} \alpha_{n, 2} x_{i-(m-1), k-[(m-1) P+m] R-\tau} \cdot \sum_{n=1}^{N_{T}} \alpha_{n, 1} \sum_{j=0}^{N-1} b_{i-j} x_{i-j, k-(j P+j+1) R} \\
& +\frac{P}{N_{T}} \sum_{k=0}^{R-1} \sum_{n=1}^{N_{T}} \alpha_{n, 2}^{2} x_{i-(m-1), k-[(m-1) P+m] R-\tau} \cdot \sum_{j=0}^{N-1} b_{i-j} x_{i-j, k-(j P+j+1) R-\tau} \\
& +\frac{P}{\sqrt{N_{T}}} \sum_{k=0}^{R-1}\left[\left(\sum_{n=1}^{N_{T}} \alpha_{n, 1} x_{i-(m-1), k-[(m-1) P+m] R}+\sum_{n=1}^{N_{T}} \alpha_{n, 2} x_{i-(m-1), k-[(m-1) P+m] R-\tau}\right) \cdot \xi_{i, k}\right] \\
& +\frac{1}{\sqrt{N_{T}}} \sum_{p=1}^{P} \sum_{k=0}^{R-1}\left[\left(\sum_{n=1}^{N_{T}} \alpha_{n, 1} \sum_{j=0}^{N-1} b_{i-j} x_{i-j, k-(j P+j+1) R}+\sum_{n=1}^{N_{T}} \alpha_{n, 2} \sum_{j=0}^{N-1} b_{i-j} x_{i-j, k-(j P+j+1) R-\tau}\right) \cdot \xi_{i-(m-1), k-[(m-1) P+m] R}\right] \\
& +\sum_{p=1}^{P} \sum_{k=0}^{R-1} \xi_{i, k} \xi_{i-(m-1), k-[(m-1) P+m] R}=A+B+C .
\end{aligned}
$$

Here,

$$
\begin{aligned}
A= & \frac{P}{N_{T}} \sum_{k=0}^{R-1} \sum_{n=1}^{N_{T}} \alpha_{n, 1}^{2} x_{i-(m-1), k-[(m-1) P+m] R} \cdot \sum_{j=0}^{N-1} b_{i-j} x_{i-j, k-(j P+j+1) R} \\
& +\frac{P}{N_{T}} \sum_{k=0}^{R-1} \sum_{n=1}^{N_{T}} \alpha_{n, 2}^{2} x_{i-(m-1), k-[(m-1) P+m] R-\tau} \cdot \sum_{j=0}^{N-1} b_{i-j} x_{i-j, k-(j P+j+1) R-\tau} \\
= & \frac{P}{N_{T}} \sum_{k=0}^{R-1} \sum_{n=1}^{N_{T}} \alpha_{n, 1}^{2} b_{i-(m-1)} x_{i-(m-1), k-[(m-1) P+m] R}^{2} \\
& +\frac{P}{N_{T}} \sum_{k=0}^{R-1} \sum_{n=1}^{N_{T}} \sum_{j=0}^{N-1} \alpha_{n, 1}^{2} b_{i-j} x_{i-(m-1), k-[(m-1) P+m] R} x_{i-j, k-(j P+j+1) R} \\
& +\frac{P}{N_{T}} \sum_{k=0}^{R-1} \sum_{n=1}^{N_{T}} \alpha_{n, 2}^{2} b_{i-(m-1)} x_{i-(m-1), k-[(m-1) P+m] R-\tau}^{2} \\
& +\frac{P}{N_{T}} \sum_{k=0}^{R-1} \sum_{n=1}^{N_{T}} \sum_{j=0}^{N-1} \alpha_{n, 2}^{2} b_{i-j} x_{i-(m-1), k-[(m-1) P+m] R-\tau} x_{i-j, k-(j P+j+1) R-\tau} .
\end{aligned}
$$

$$
\begin{array}{r}
B=\frac{P}{\sqrt{N_{T}}} \sum_{k=0}^{R-1}\left[\left(\sum_{n=1}^{N_{T}} \alpha_{n, 1} x_{i-(m-1), k-[(m-1) P+m] R}\right.\right. \\
\left.\left.+\sum_{n=1}^{N_{T}} \alpha_{n, 2} x_{i-(m-1), k-[(m-1) P+m] R-\tau}\right) \cdot \xi_{i, k}\right] \\
+\frac{1}{\sqrt{N_{T}}} \sum_{p=1}^{P} \sum_{k=0}^{R-1}\left[\left(\sum_{n=1}^{N_{T}} \alpha_{n, 1} \sum_{j=0}^{N-1} b_{i-j} x_{i-j, k-(j P+j+1) R}\right.\right. \\
\left.+\sum_{n=1}^{N_{T}} \alpha_{n, 2} \sum_{j=0}^{N-1} b_{i-j} x_{i-j, k-(j P+j+1) R-\tau}\right) \\
\left.\cdot \xi_{i-(m-1), k-[(m-1) P+m] R}\right] .
\end{array}
$$

$$
C=\sum_{p=1}^{P} \sum_{k=0}^{R-1} \xi_{i, k} \xi_{i-(m-1), k-[(m-1) P+m] R} .
$$

Since the sidelobe of the chaotic map is zero, (14) can be simplified to

$$
\begin{aligned}
A= & \frac{P}{N_{T}} \sum_{k=0}^{R-1} \sum_{n=1}^{N_{T}} \alpha_{n, 1}^{2} b_{i-(m-1)} x_{i-(m-1), k-[(m-1) P+m] R}^{2} \\
& +\frac{P}{N_{T}} \sum_{k=0}^{R-1} \sum_{n=1}^{N_{T}} \alpha_{n, 2}^{2} b_{i-(m-1)} x_{i-(m-1), k-[(m-1) P+m] R-\tau}^{2}
\end{aligned}
$$

The information bit $\widehat{b}_{i-(m-1)}$ can be obtained by (13):

$$
\widehat{b}_{i-(m-1)}= \begin{cases}+1 & Z_{i-(m-1)}>0, \\ -1 & Z_{i-(m-1)} \leq 0 .\end{cases}
$$

From the above description, it can be concluded that the terms of the decision variable in (14), (15), and (16) can be approximated as obeying the Gaussian distribution. In (14), (15), and (16), the calculation of the mean and the variance are given by

$$
\begin{aligned}
E[A] & =\frac{P R}{N_{T}} b_{i-(m-1)}\left[\left(\sum_{n=1}^{N_{T}} \alpha_{n, 1}\right)^{2}+\left(\sum_{n=1}^{N_{T}} \alpha_{n, 2}\right)^{2}\right], \\
E[B] & =E[C]=0, \\
\operatorname{var}[A] & =\frac{P^{2} R}{N_{T}^{2}}(N-1)\left[\left(\sum_{n=1}^{N_{T}} \alpha_{n, 1}\right)^{4}+\left(\sum_{n=1}^{N_{T}} \alpha_{n, 2}\right)^{4}\right],
\end{aligned}
$$




$$
\begin{aligned}
\operatorname{var}[B]= & \frac{P^{2} R N_{0}}{2 N_{T}}\left[\left(\sum_{n=1}^{N_{T}} \alpha_{n, 1}\right)^{2}+\left(\sum_{n=1}^{N_{T}} \alpha_{n, 2}\right)^{2}\right] \\
& +\frac{P R N_{0}}{2 N_{T}}\left[\left(\sum_{n=1}^{N_{T}} \alpha_{n, 1}\right)^{2}+\left(\sum_{n=1}^{N_{T}} \alpha_{n, 2}\right)^{2}\right], \\
\operatorname{var}[C]= & \frac{P R N_{0}^{2}}{4}
\end{aligned}
$$

The mean and variance that can be obtained by (19), (20), (21), (22), and (23) are

$$
\begin{aligned}
E\left[Z_{i-(m-1)}\right]= & E[A]+E[B]+E[C] \\
= & \frac{P R}{N_{T}} b_{i-(m-1)}\left[\left(\sum_{n=1}^{N_{T}} \alpha_{n, 1}\right)^{2}+\left(\sum_{n=1}^{N_{T}} \alpha_{n, 2}\right)^{2}\right] \\
\operatorname{var}\left[Z_{i-(m-1)}\right]= & \operatorname{var}[A]+\operatorname{var}[B]+\operatorname{var}[C] \\
= & \frac{P^{2} R}{N_{T}^{2}}(N-1)\left[\left(\sum_{n=1}^{N_{T}} \alpha_{n, 1}\right)^{4}+\left(\sum_{n=1}^{N_{T}} \alpha_{n, 2}\right)^{4}\right] \\
& +\frac{P^{2} R N_{0}}{2 N_{T}}\left[\left(\sum_{n=1}^{N_{T}} \alpha_{n, 1}\right)^{2}+\left(\sum_{n=1}^{N_{T}} \alpha_{n, 2}\right)^{2}\right] \\
& +\frac{P R N_{0}}{2 N_{T}}\left[\left(\sum_{n=1}^{N_{T}} \alpha_{n, 1}\right)^{2}+\left(\sum_{n=1}^{N_{T}} \alpha_{n, 2}\right)^{2}\right] \\
& +\frac{P R N_{0}^{2}}{4} .
\end{aligned}
$$

The BER of the system can be expressed as

$$
\begin{aligned}
\operatorname{BER}= & \frac{1}{2} \operatorname{prob}\left(Z_{i-(m-1)}<0 \mid b_{i-(m-1)}=+1\right) \\
& +\frac{1}{2} \operatorname{prob}\left(Z_{i-(m-1)}>0 \mid b_{i-(m-1)}=-1\right) \\
= & \frac{1}{2} \operatorname{erfc}\left(\frac{\left|E\left[Z_{i-(m-1)}\right]\right|}{\sqrt{2 \operatorname{var}\left[Z_{i-(m-1)}\right]}}\right),
\end{aligned}
$$

where erfc $(\cdot)$ represents a complementary error function, $\operatorname{erfc}(x) \equiv 2 / \sqrt{\pi} \int_{x}^{\infty} e^{-\mu^{2}} d \mu$.

The BER of the MISO-SRMR-DCSK system can be expressed as

$$
\operatorname{BER}\left(\gamma_{b}\right)=\frac{1}{2} \operatorname{erfc}\left(\sqrt{\frac{2(N-1) \Lambda_{2}}{R \Lambda_{1}^{2}}+\frac{(N+P) \Gamma}{\gamma_{b}}+\frac{\beta \Gamma^{2}}{2 \gamma_{b}^{2}}}\right)^{-1}
$$

Here,

$$
\begin{aligned}
& \Lambda_{1}=\left[\left(\sum_{n=1}^{N_{T}} \alpha_{n, 1}\right)^{2}+\left(\sum_{n=1}^{N_{T}} \alpha_{n, 2}\right)^{2}\right] \text {, } \\
& \Lambda_{2}=\left[\left(\sum_{n=1}^{N_{T}} \alpha_{n, 1}\right)^{4}+\left(\sum_{n=1}^{N_{T}} \alpha_{n, 2}\right)^{4}\right] \text {, } \\
& \Gamma=1+\frac{1}{P N}, \\
& \gamma_{b}=\underbrace{\frac{\left(\sum_{n=1}^{N_{T}} \alpha_{n, 1}\right)^{2} E_{b}}{N_{T} N_{0}}}_{\gamma_{1}}+\underbrace{\frac{\left(\sum_{n=1}^{N_{T}} \alpha_{n, 2}\right)^{2} E_{b}}{N_{T} N_{0}}}_{\gamma_{2}} .
\end{aligned}
$$

$\overline{\gamma_{1}}=E\left(\gamma_{1}\right)$ and $\overline{\gamma_{2}}=E\left(\gamma_{2}\right)$ represent the average signalto-noise ratio for each path. The PDF of $\gamma_{b}$ is given by

$$
f\left(\gamma_{b}\right)= \begin{cases}\frac{\gamma_{b}}{\bar{\gamma}^{2}} e^{-\gamma_{b} / \overline{\gamma_{1}}}, & E\left(\alpha_{1}^{2}\right)=E\left(\alpha_{2}^{2}\right), \\ \frac{e^{-\gamma_{b} / \overline{\gamma_{1}}}-e^{-\gamma_{b} / \overline{\gamma_{2}}}}{\overline{\gamma_{1}}-\overline{\gamma_{2}}}, & E\left(\alpha_{1}^{2}\right) \neq E\left(\alpha_{2}^{2}\right),\end{cases}
$$

From formulas (26) and (28), the BER formula of the MISO-SRMR-DCSK system in the multipath Rayleigh fading channel is as follows

$$
\operatorname{BER}=\int_{0}^{\infty} \operatorname{BER}\left(\gamma_{b}\right) f\left(\gamma_{b}\right) \mathrm{d} \gamma_{b}
$$

In the channel parameters $\alpha_{n, 1}=1$ and $\alpha_{n, 2}=0$, the BER of the MISO-SRMR-DCSK system under the AWGN channel can be expressed as

$$
\mathrm{BER}=\frac{1}{2} \operatorname{erfc}\left(\sqrt{\frac{2(N-1)}{R}+\frac{\Gamma(N+P) N_{0}}{2 E_{b}}+\frac{\beta \Gamma^{2} N_{0}^{2}}{8 E_{b}^{2}}}\right)^{-1} .
$$

The effect of the reference signal length on the bit error rate and the derivation of the optimal reference signal length corresponding to the minimum BER will be analyzed in this part.

Firstly, we define $f(R)$ from (30) as

$$
f(R)=\frac{2(N-1)}{R}+\frac{\Gamma(N+P) N_{0}}{2 E_{b}}+\frac{\beta \Gamma^{2} N_{0}^{2}}{8 E_{b}^{2}} .
$$

Next,

$$
\frac{d f(R)}{d R}=-\frac{2(N-1)}{R^{2}}+\frac{P \Gamma^{2}}{8}\left(\frac{E_{b}}{N_{0}}\right)^{-2} .
$$

Then,

$$
\frac{2(N-1)}{R^{2}}-\frac{P \Gamma^{2}}{8}\left(\frac{E_{b}}{N_{0}}\right)^{-2}=0 .
$$




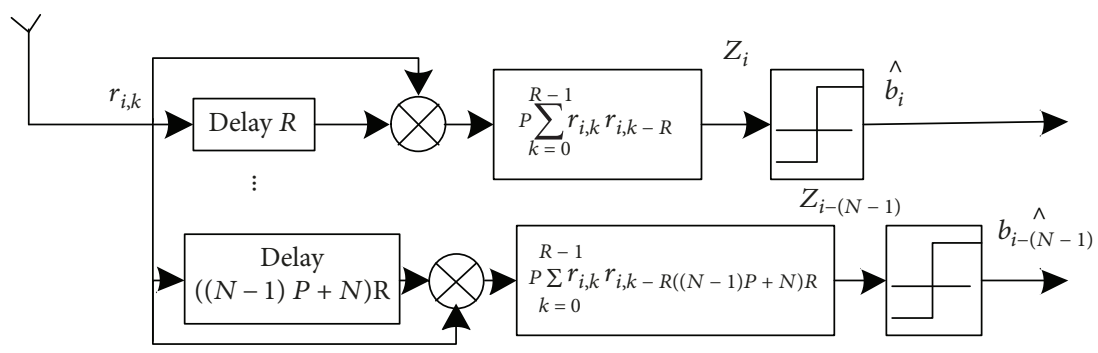

FIGURE 6: SISO-SRMR-DCSK receiver.

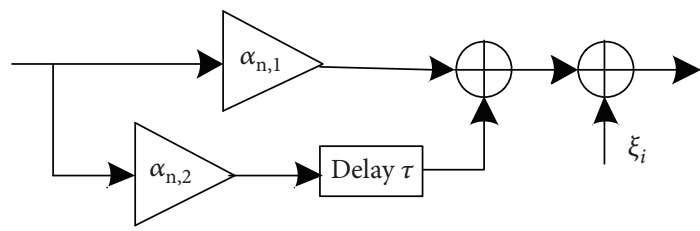

FIGURE 7: Two-ray Rayleigh quasi-static block faded channel model.
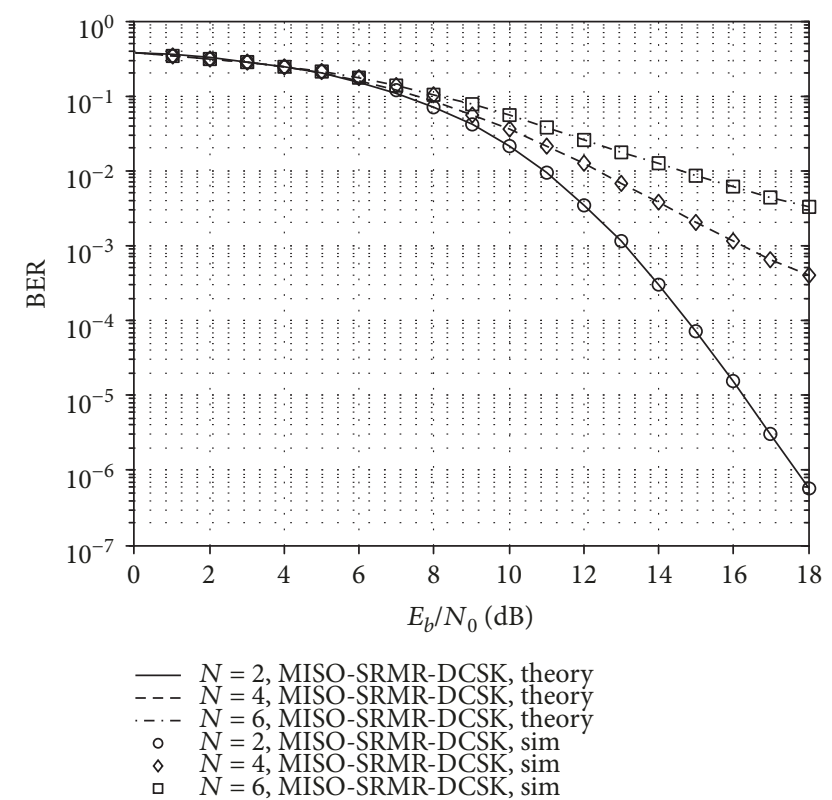

FIGURE 8: Simulation and analytical BER performance of MISOSRMR-DCSK in AWGN channels for $\beta=100, P=2$, and various $N$.

Finally, the solution for (33) is the minimizing solution

$$
R_{\mathrm{opt}}=\sqrt{\frac{N-1}{P}} \times \frac{4 E_{b}}{\Gamma N_{0}} .
$$

\section{Simulation}

In order to validate the BER performance of the MISOSRMR-DCSK scheme and compare it with SR-DCSK and HE-DCSK, the theoretic BER expressions are verified and well justified by simulation results under AWGN and

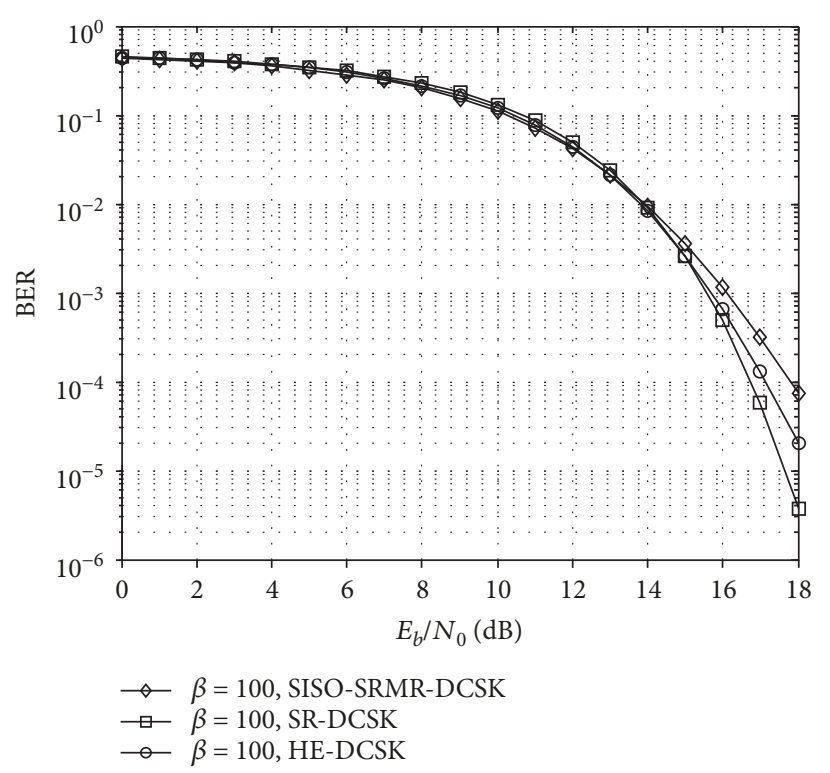

FIGURE 9: BER performance of SISO-SRMR-DCSK, SR-DCSK, and HE-DCSK for $\beta=100, P=2$, and $N=2$ in AWGN channels.

multipath fading channels. In the next part, the simulation diagram will be introduced and explained.

4.1. AWGN Channel. In this part, we will compare the analytical expression of the BER performance in the AWGN channel to simulation results. From Figure 8, we can clearly see that the theoretic BER expression and simulation curves are a perfect match in the AWGN channel. The result proves the correctness of the theoretical derivation. Figure $8 \mathrm{com}$ pares the simulated BER against SNR in the AWGN channel for different values of other character. Values of $N=2,4$, and 6 transmit a different number of bits. However, as the number of transmission bits increases, the BER performance of the system is gradually deteriorating, which is due to intersymbol interference. Figure 9 compares the simulated BER against $E_{b} / N_{0}$ of SR-DCSK, HE-DCSK, and SISOSRMR-DCSK in the AWGN channel for $\beta=100, N=2$, where the HE-DCSK [13] system is the SRMR-DCSK system in the case of $P=1$ and $N=2$. From the simulation curves, we can see that the SR-DCSK [6] system has better BER performance than the classical systems HE-DCSK and SISO-SRMR-DCSK. The MISO-SRMR-DCSK system has a higher transmission rate and a lower BER performance than the SR-DCSK and HE-DCSK systems. And when 


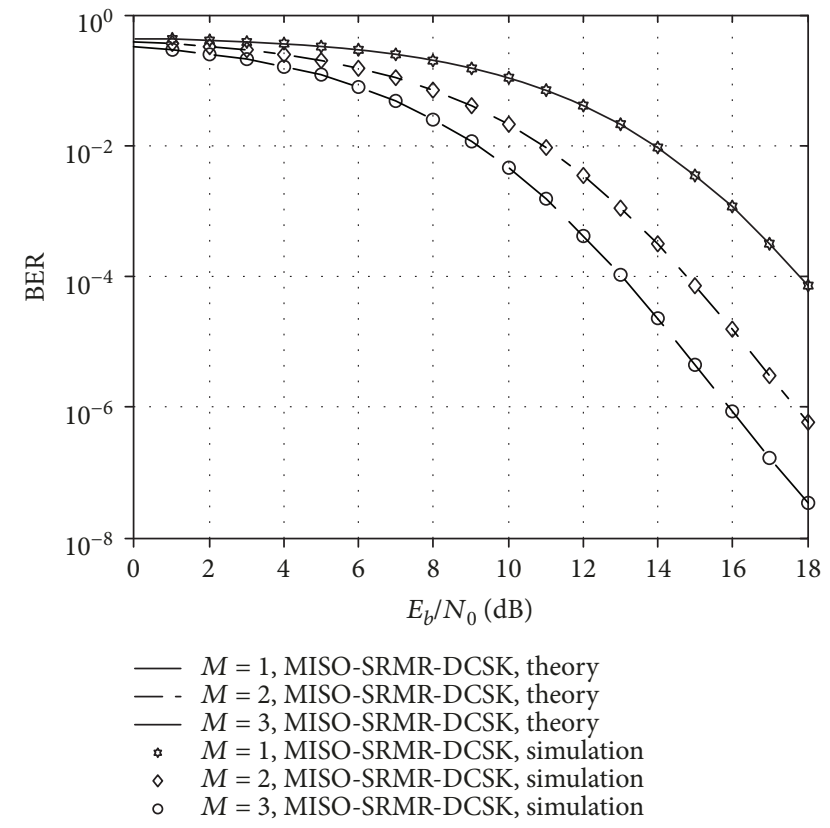

Figure 10: BER performance of MISO-SRMR-DCSK for $\beta=100$, $P=2, M=1,2,3$, and $N=2$ in AWGN channels.

$N=1$, the SRMR-DCSK system is the SR-DCSK system; when $N=2$ and $\beta=R$, the SISO-SRMR-DCSK system is the HE-DCSK system, under the same conditions to increase the number of transmission antennas to improve the original system BER performance. Figure 10 shows the BER performance of the system with different transmission antennas. From Figure 10, we can see that the performance of the system is gradually optimized with the increase in the number of transmission antennas.

The effect of the reference signal length $R$ on the performance of MISO-SRMR-DCSK for $N=2$ in AWGN channels is analyzed, and the simulation results are displayed in Figure 11. In Figure 11, different SNRs almost show the same trend; that is to say, with the increase in the length of the reference signal, BER performance is gradually increasing. In Figure 12, we can see that the optimal reference signal length is related to $E_{b} / N_{0}, N$, and $P$. In (33), $E_{b} / N_{0}, N$, and $P$ are fixed, and the optimal reference sequence length at this time can be obtained. As shown in Figure 12, in the case of $E_{b} / N_{0}=15 \mathrm{~dB}, N=2$, and $P=2$, it can be concluded that the optimal sequence length under this condition is 71.5 , and the result is rounded to a final result of 72 , because the sequence length is an integer. It is found that under the above conditions, the BER performance of the system is best at $15 \mathrm{~dB}$ when the reference sequence length is 72 .

4.2. Rayleigh Fading Channel. In this part, we will compare the analytical expression of the BER performance in the Rayleigh fading channel to simulation results in the two following cases:

Case 1. The average channel gain of the first channel is equal to the average channel gain of the second channel $E\left[\alpha_{n, 1}^{2}\right]=1 / 2$ and $E\left[\alpha_{n, 2}^{2}\right]=1 / 2$.

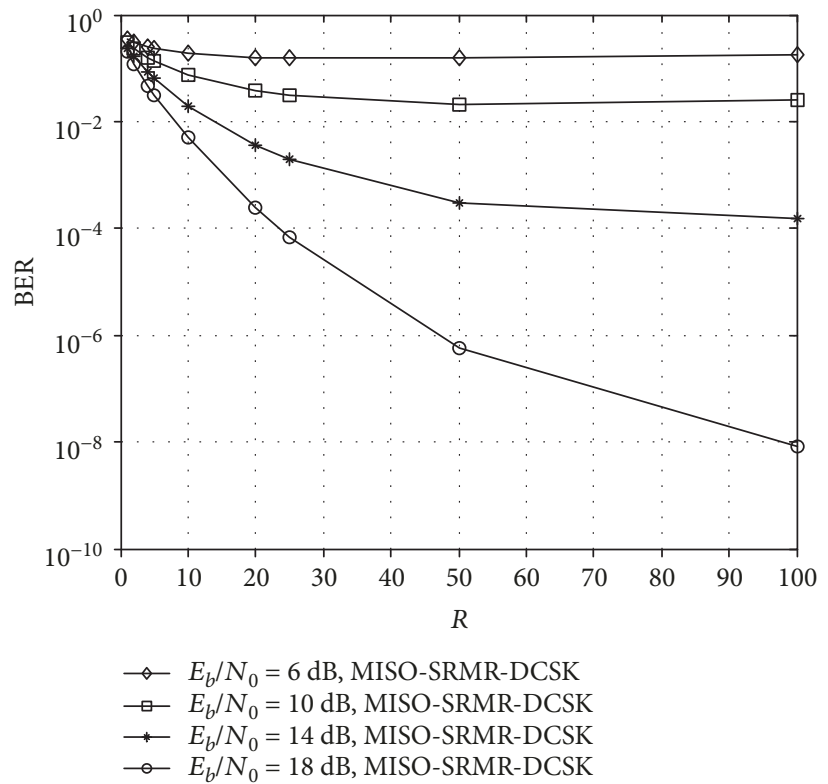

FIgURE 11: Simulation of the BER for a data signal length of $\beta=100$ versus different values of $R$ over the AWGN channel.

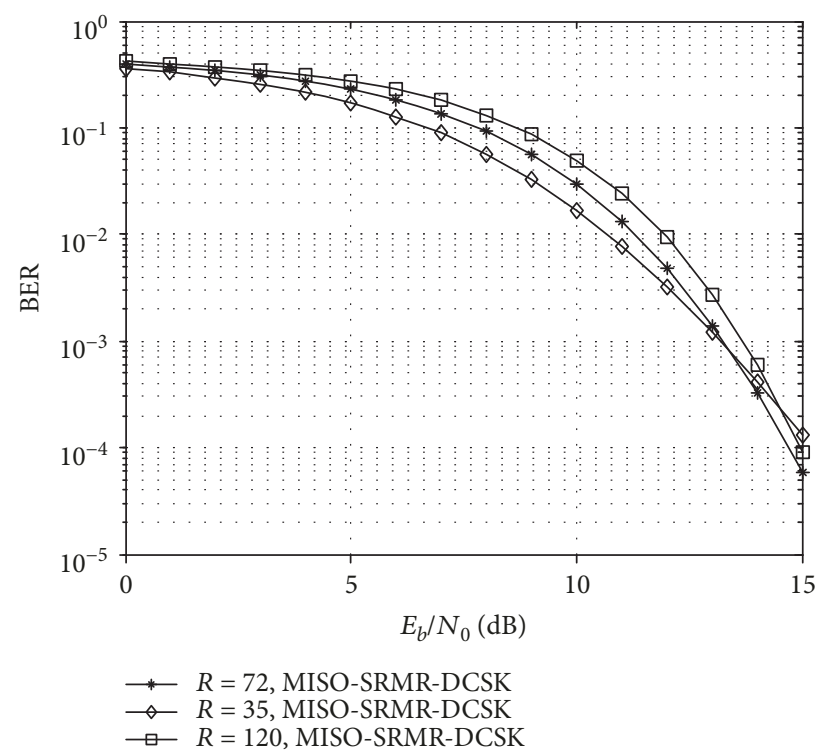

FIGURE 12: Simulation of the BER performance for the effect of optimal reference sequence length and general reference signal length of MISO-SRMR-DCSK in the AWGN channel for $E_{b} / N_{0}=$ $15 \mathrm{~dB}, N=2$, and $P=2$.

Case 2. The average channel gain of the first channel is $3 \mathrm{~dB}$ higher than the average channel gain of the second channel $E\left[\alpha_{n, 1}^{2}\right]=2 / 3$ and $E\left[\alpha_{n, 2}^{2}\right]=1 / 3$.

In the simulation, we assume that the delay of the multipath channel is $\tau_{n, 1}=0$ and $\tau_{n, 2}=\tau$, and the channel delay is much smaller than the chaotic sequence length $\beta$, that is, $0<\tau \ll \beta$. In the actual simulation, $\tau$ will be similar to 0 processing. Figure 13 compares the simulated BER 

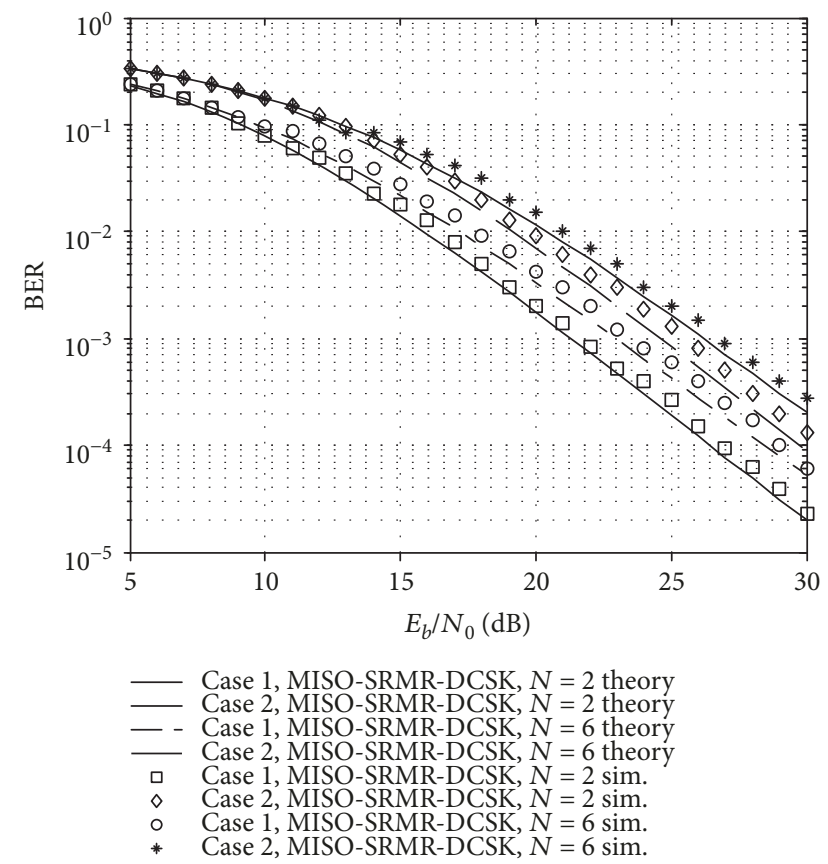

FIGURE 13: Simulation and analytical BER performance of MISOSRMR-DCSK in Rayleigh fading channels for $\beta=100, P=2$, and various $N$.

against different channel gains in the Rayleigh fading channel for different values of $N$. At the same transmission rate, for Case 1, the receiver receives two versions of the same signal with the same average power gain. Consequently, each path contributes significantly to the decision, but for Case 2, where there is a dominant path. From the simulation results obtained, we can say that increasing diversity allows combating the effect of the multipath Rayleigh fading channel. At the same transmission rate, the performance of the same channel gain of the two channels is about $3 \mathrm{~dB}$ higher than that of the different channel gain.

Figure 14 shows the contrast between the theoretical BER and the simulation results for $\beta=100,500$ and $N=2$ in the Rayleigh fading channel. As can be seen from Figure 14, the BER performance at $\beta=500$ is always inferior to that of $\beta=100$ when the channel gain is the same or different.

\section{Conclusion}

In the paper, MISO-SRMR-DCSK is introduced and analyzed. This system on the basis of the original DCSK system improves the data transmission rate and reduces the bit transmitted power. At the transmitter, two antennas are used to transmit data, in which the length of the reference signal in each frame of data is shortened to $1 / P$ of the original DCSK system, and the data slot repeats by $P$ times of reference slots and transmits $N$-bit data. The receiver uses an antenna to receive the signal, the received signal is delayed for different times, and the data signal $P$ times the correlation operation, the final operation results, and the decision threshold comparison. In this paper, the theoretical BER
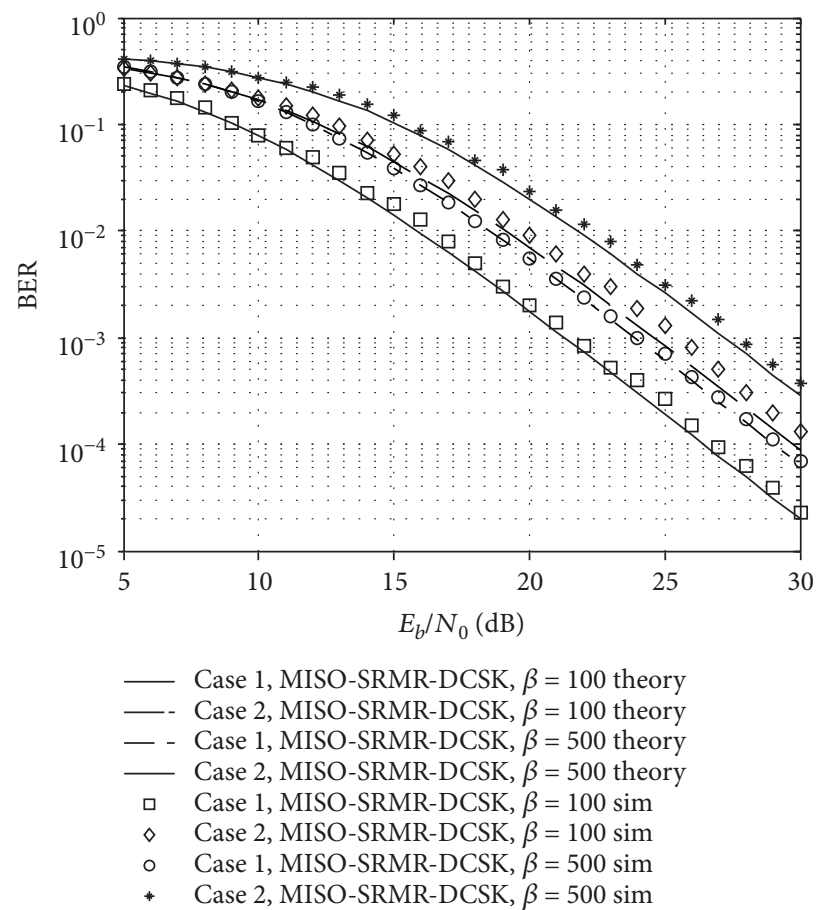

FIGURE 14: Simulation and analytical BER performance of MISOSRMR-DCSK in Rayleigh fading channels for $N=2, P=2$, and various $\beta$.

performance of the Gaussian channel and the two-ray Rayleigh fading channel is analyzed by Gaussian approximation, and the correctness of the system is verified by Monte Carlo simulation. In the AWGN channel, the performance of the MISO-SRMR-DCSK system is gradually optimized with the increase in the number of transmission antennas. At the same time, the system BER performance under the best reference sequence length is verified and analyzed. In the two-ray Rayleigh fading channel, for the system in different reference sequence length is analyzed, respectively. The simulation results show that the BER performance of MISO-SRMR-DCSK gradually deteriorated due to the increase in the intersymbol interference with the increase in the number of transmission bits under the same conditions.

For the complexity of the system, the MISO-SRMRDCSK system requires multiple transmission antennas, but the system improves spectrum utilization and transmission rates, so the complexity of the sacrifice system is worthwhile.

\section{Data Availability}

The data used to support the findings of this study are available from the corresponding author upon request.

\section{Conflicts of Interest}

The authors declare that they have no conflicts of interest. 


\section{Acknowledgments}

This work was supported in part by the National Natural Science Foundation of China under Grant 61771085 and 61371164, and in part by the Research Project of Chongqing Educational Commission under Grant KJ1600427 as well as by the Chongqing Distinguished Youth Foundation under Grant CSTC2009CA2003.

\section{References}

[1] H. Dedieu, M. P. Kennedy, and M. Hasler, "Chaos shift keying: modulation and demodulation of a chaotic carrier using selfsynchronizing Chua's circuits," IEEE Transactions on Circuits and Systems II: Analog and Digital Signal Processing, vol. 40, no. 10, pp. 634-642, 1993.

[2] G. Kolumbán, B. Vizvári, W. Schwarz, and A. Abel, "Differential chaos shift keying: a robust coding for chaos communication," in NDES'96, pp. 92-97, Seville, Spain, 1996.

[3] M. Kavehrad and B. Ramamurthi, "Direct-sequence spread spectrum with DPSK modulation and diversity for indoor wireless communications," IEEE Transactions on Communications, vol. 35, no. 2, pp. 224-236, 1987.

[4] M. Herceg, K. Miličević, and T. Matić, "Correlation-multidelay-shift-keying for chaos based communications," Wireless Personal Communications, vol. 88, no. 2, pp. 283-294, 2016.

[5] Z. Galias and G. M. Maggio, "Quadrature chaos-shift keying: theory and performance analysis," IEEE Transactions on Circuits and Systems I: Fundamental Theory and Applications, vol. 48, no. 12, pp. 1510-1519, 2001.

[6] G. Kaddoum, E. Soujeri, and Y. Nijsure, "Design of a short reference noncoherent chaos-based communication systems," IEEE Transactions on Communications, vol. 64, no. 2, pp. 680$689,2016$.

[7] G. Cai, L. Wang, and T. Huang, "Channel capacity of M-ary differential chaos shift keying modulation over AWGN channel," in 2013 13th International Symposium on Communications and Information Technologies (ISCIT), pp. 91-95, Surat Thani, Thailand, 2013.

[8] P. Chen, L. Wang, and G. Chen, "DDCSK-Walsh coding: a reliable chaotic modulation-based transmission technique," IEEE Transactions on Circuits and Systems II: Express Briefs, vol. 59, no. 2, pp. 128-132, 2012.

[9] G. Kaddoum, F. Gagnon, and F. D. Richardson, "Design of a secure multi-carrier DCSK system," in 2012 International Symposium on Wireless Communication Systems (ISWCS), pp. 964-968, Paris, France, 2012.

[10] G. Kaddoum, F. D. Richardson, and F. Gagnon, "Design and analysis of a multi-carrier differential chaos shift keying communication system," IEEE Transactions on Communications, vol. 61, no. 8, pp. 3281-3291, 2013.

[11] W. K. Xu, L. Wang, and G. Kolumbán, "A novel differential chaos shift keying modulation scheme," International Journal of Bifurcation and Chaos, vol. 21, no. 3, pp. 799-814, 2011.

[12] G. Kaddoum and F. Gagnon, "Design of a high-data-rate differential chaos-shift keying system," IEEE Transactions on Circuits and Systems II: Express Briefs, vol. 59, no. 7, pp. 448452, 2012.

[13] H. Yang and G. P. Jiang, "High-efficiency differentialchaos-shift-keying scheme for chaos-based noncoherent communication," IEEE Transactions on Circuits and Systems II: Express Briefs, vol. 59, no. 5, pp. 312-316, 2012.

[14] H. Yang and G. P. Jiang, "Reference-modulated DCSK: a novel chaotic communication scheme," IEEE Transactions on Circuits and Systems II: Express Briefs, vol. 60, no. 4, pp. 232-236, 2013.

[15] F. C. M. Lau, K. Y. Cheong, and C. K. Tse, "Permutation-based DCSK and multiple access DCSK systems," IEEE Transactions on Circuits and Systems I: Fundamental Theory and Applications, vol. 50, no. 6, pp. 733-742, 2003. 


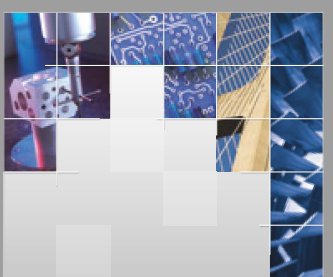

\section{Enfincering}
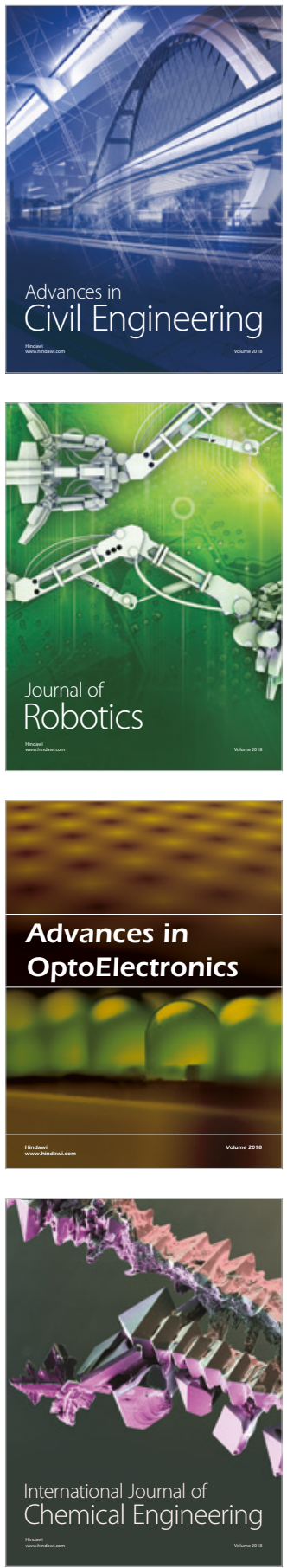

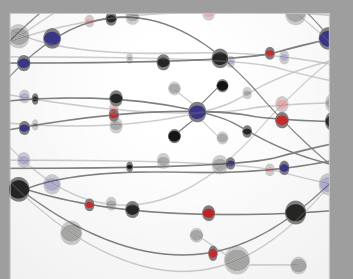

\section{Rotating \\ Machinery}

The Scientific World Journal

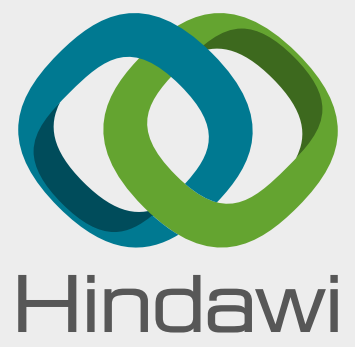

Submit your manuscripts at

www.hindawi.com
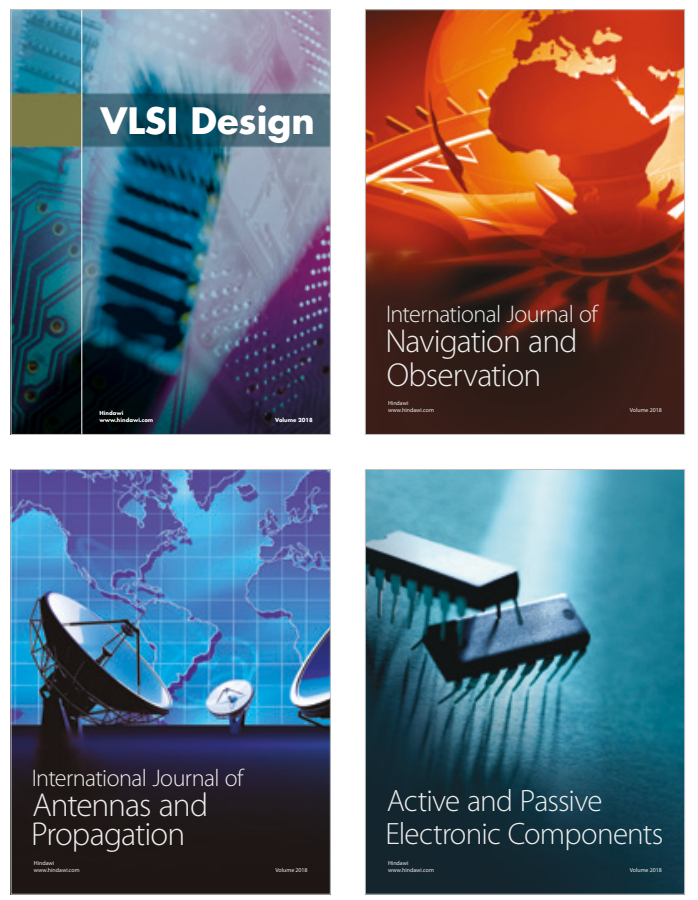
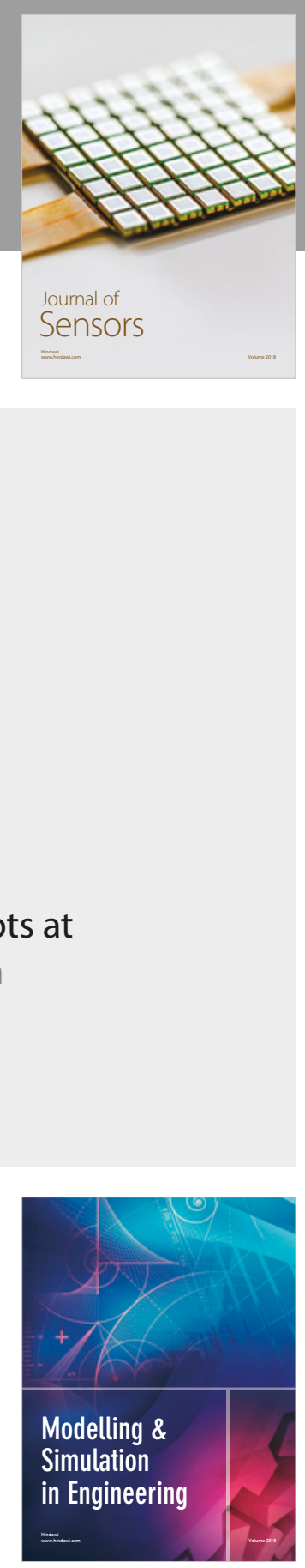

\section{Advances \\ Multimedia}
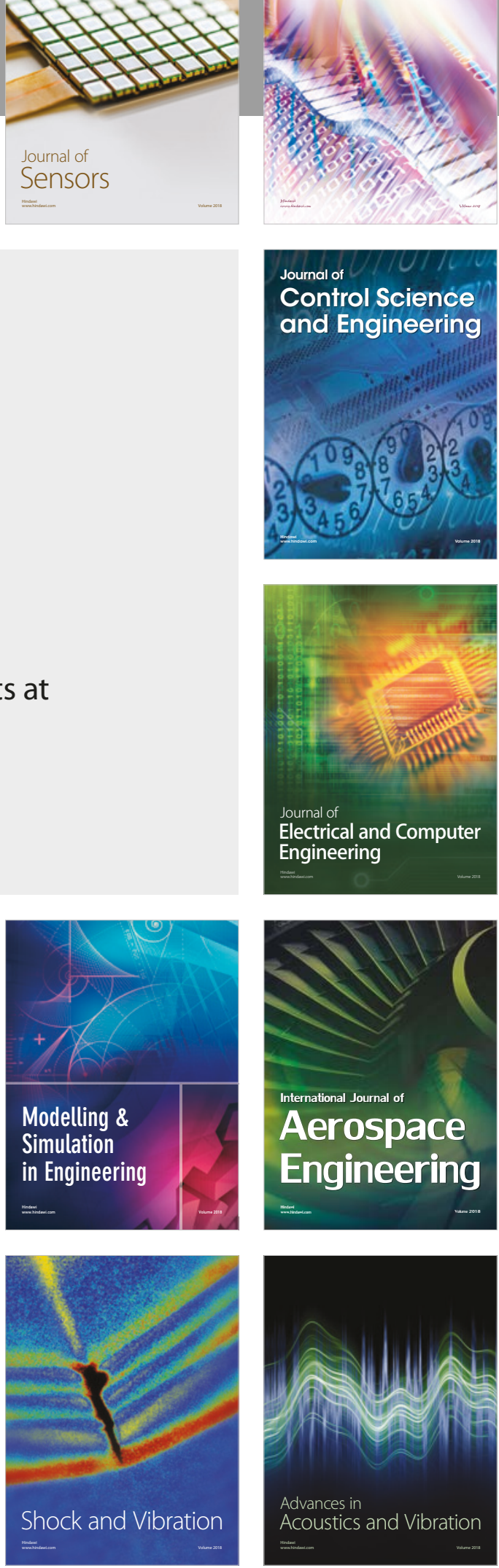